\title{
Analyse discursive des incises de citation
}

\author{
Laurence Danlos $^{1}$, Benoît Sagot ${ }^{1}$ et Rosa Stern ${ }^{1,2}$ \\ 1. Alpage, INRIA Paris-Rocquencourt \& Université Paris 7 \\ 30 rue du Château des Rentiers, 75013 Paris, France \\ 2. Agence France-Presse - Medialab \\ 2 place de la Bourse, 75002 Paris, France \\ laurence.danlos@linguist.jussieu.fr, benoit.sagot@inria.fr, rosa.stern@afp.com
}

\section{Introduction}

Qui a dit quoi ? A qui attribue-t-on quel propos? Ces questions sont cruciales dans de nombreuses applications du TAL, en particulier dans les systèmes d'extraction d'information à partir de corpus journalistiques qui comportent de nombreux discours rapportés et citations.

Il existe des formes variées en français pour attribuer des propos à une source autre que le locuteur. Citons parmi d'autres : le discours indirect introduit par un verbe de discours rapporté (1a); le discours direct comprenant une citation et une incise de citation (1b); l'insertion d'un syntagme prépositionnel introduit principalement par les préposition selon ou d'après (1c). Dans ces exemples, le segment attributif est souligné, ainsi que les signes de ponctuation l'encadrant éventuellement. De ce fait, en supprimant les éléments soulignés de la phrase, il ne reste que le segment attribué, à savoir les impôts vont baisser dès le mois prochain, qui est encadré de guillemets en (1b).

(1) a. Le député a dit que les impôts vont baisser dès le mois prochain.

b. "Les impôts vont baisser dès le mois prochain", a dit le député.

c. Les impôts vont baisser dès le mois prochain, selon/d'après le député.

Dans cet article, nous nous concentrons sur des exemples comme (1b), c'est-à-dire des exemples où le segment attributif est une incise de citation, le segment attribué une citation, repérable par la présence de guillemets, typographiquement représentés par les signes " et ". Nous appelons verbes de citation les verbes qui peuvent apparaître comme têtes verbales des incises de citation. Pour des raisons qui deviendront évidentes par la suite nous qualifions des énoncés comme (1b) de Discours avec Incise de Citation (abrégés en $D I C)$.

La citation et l'incise de citation forment un agrégat soudé dans la mesure où une incise de citation demande la présence d'une citation. La position que nous défendons est que cet agrégat relève principalement du niveau sémantico-discursif et non du niveau syntaxico-phrastique. Plus précisément, notre position est qu'il n'existe pas de lien argumental syntaxique entre le verbe de citation et la citation, bien qu'il soit généralement considéré dans la littérature que la citation correspond à l'objet direct du verbe de citation, (Bonami \& Godard, 2007 ; Lamiroy \& Charolles, 2008). Un DIC respecte certaines contraintes syntaxiques (par exemple, sur la position de l'incise par rapport à la citation), mais il respecte surtout des contraintes sémantiques que l'on doit représenter au niveau discursif. Cette position vient d'une étude de corpus de dépêches de l'AFP (Agence France Presse) dont nous avons extrait une liste de 232 verbes de citation, dont seule la moitié peut être employée comme verbes de discours rapporté analogues à dire. L'autre moitié est composée 
de verbes intransitifs, mais également de verbes transitifs comme commenter, dont l'objet direct est sans rapport (ni syntaxique ni sémantique) avec la citation. La description de ces derniers verbes nécessite deux cadres de sous-catégorisation, l'un au niveau syntaxico-phrastique, l'autre au niveau sémantico-discursif. Nous montrerons que cette solution s'impose aussi pour les verbes de discours rapporté employés comme verbes de citation et pour les verbes intransitifs.

Cet article est structuré de la façon suivante. La Section 2 rappelle brièvement les principales propriétés des citations et des incises de citation. La Section 3 étudie en détail les trois classes syntaxiques de verbes de citation que nous avons identifiées grâce au corpus de l'AFP, et défend l'existence de deux cadres de souscatégorisation pour les verbes de citation, l'un au niveau syntaxico-phrastique, l'autre au niveau sémanticodiscursif. Le premier est examiné à la Section 4 - dans le cadre du lexique Lefff qui est utilisé dans différentes applications de TAL —, le second à la Section 5. Cet article repose à la fois sur des exemples fabriqués et sur des exemples de corpus, notamment des exemples du corpus de l'AFP.

\section{Bref rappel sur les citations et les incises de citation}

Les citations font l'objet d'une vaste littérature tant en philosophie qu'en linguistique. Nous nous contentons ici de rappeler brièvement leurs caractéristiques principales, résumées par exemple par Maier (2009) :

- une citation peut être dans une langue différente de celle employée dans le segment attributif (2a);

- une citation peut être formée d'un texte de plusieurs phrases (2b);

- une citation dénote a priori tout type d'acte de langage (assertion, interrogation, exclamation), mais nous verrons toutefois que le type d'acte de langage de la citation est contraint par la tête lexicale du segment attributif ;

- une citation est référentiellement opaque ;

- l'interprétation des indexicaux est particulière dans la mesure où, par exemple, le pronom je dans une citation ne réfère pas au locuteur mais à la source de la citation $(2 \mathrm{~b})$;

- l'attribution d'un «nom de qualité » (cet imbécile) n'est pas du cru du locuteur dans une citation, mais de la source de la citation (2c).

(2) a. "Ich bin ein Berliner", lance le président américain John Kennedy en visite à Berlin (...) [LeMatin.ch du 03/11/09]

b. "J'ai trouvé (sa) colère suspecte et préparée. Je suis outré par ce que ce qui a été dit, c'est des mensonges", a-t-il ajouté [AFP, dépêche TX-SGE-UXM34 du 03/05/07]

c. "Cet imbécile est timide", a dit Fred.

Tournons-nous maintenant vers les incises de citation. Leur caractéristique principale (en français soutenu) est l'inversion obligatoire du sujet, que celui-ci soit réalisé sous forme d'un GN ou d'un clitique (commenta le député, commenta-t-il). Cette inversion du sujet s'accompagne de contraintes sur la possibilité de maintenir les éventuels autres compléments sous-catégorisés par le verbe. On peut ainsi noter l'inacceptabilité d'incise de citation telles que *commenta-t-il la décision ou *commenta-t-il par une remarque acerbe, mais l'acceptabilité de dit-il au président de séance ou de fulmina-t-il contre ce point. Ces contraintes seront examinées en détail à la Section 3, puis modélisées à la Section 4. À l'inverse, il n'existe à notre connaissance aucune contrainte concernant les ajouts (annonça-t-il après le dîner) et les modaux ou auxiliaires modifiant la tête lexicale de l'incise de citation (devrait-il avoir dit). Toutefois, une incise de citation ne se trouve pas sous la portée d'une négation (*n'a pas dit le député), sauf pour des verbes comme cacher ou taire $(\approx$ ne pas dire) pour lequel la négation est obligatoire (3) (de Cornulier, 1978). 
(3) 'On peut d'ores et déjà, compte tenu de ce qui se passe dans le reste du monde, dire qu'il y aura plusieurs millions de Français qui seront concernés par cette grippe", n’a pas caché le Premier ministre. [Le Point.fr du 24/07/2009]

Examinons la position des incises de citation. En (1b), l'incise est en « position finale » : elle apparaît après la citation. Mais l'incise peut tout aussi bien se situer en « position médiane », c'est-à-dire à l'intérieur de la citation (4a), tout en respectant les positions possibles des ajouts incidents (Bonami \& Godard, 2007). À notre connaissance, la position médiane d'une incise n'induit aucune différence par rapport à la position finale, et ce, tant sur le plan lexical, syntaxique, sémantique que discursif. Par contre, il existe une forte restriction sur la « position initiale » d'une incise de citation, c'est-à-dire avant la citation : ce cas de figure ne s'observe que lorsque l'incise est précédée d'un connecteur de discours, voir (4b) dans lequel nous avons mis le connecteur en caractères gras et le contexte gauche du connecteur en italique.

(4) a. "Les impôts vont, dit le député, baisser dès le mois prochain".

b. Pierre nous a annoncé son arrivée et, a-t-il précisé, "j' arriverai avec une fiancée"

Nous concluons cette brève présentation des citations et des incises de citation par la remarque suivante : l'agrégat formé par une citation et son incise de citation ne peut être enchâssé ni sous un verbe à complément phrastique (5a), ni sous une une subordonnée introduite par une conjonction de subordination (5b).

(5) a. *Je crois que "donne-moi la main !” a lancé Paul à Marie (Bonami \& Godard, 2008)

b. *Je suis furieuse parce que, “Tu es paresseuse", m'a dit Fred.

Cette interdiction d'enchâssement, qui pèse aussi sur les discours formés de plusieurs phrases, est un argument fort pour défendre l'hypothèse que l'agrégat formé par une citation et son incise de citation relève du niveau discursif et non du niveau phrastique. L'analyse lexicale présentée ci-dessous va présenter d'autres arguments en faveur de cette hypothèse.

\section{Verbes de citation}

Nous avons mené une analyse semi-automatique des incises de citation dans un corpus de l'AFP afin d'établir une liste de verbes qui peuvent être la tête d'une incise de citation (Sagot et al., 2010). Ce travail a débouché sur une liste (non exhaustive) de 232 verbes que nous avons répartis en trois classes :

- Classe 1 : 118 verbes transitifs de discours rapporté comme dire ou déclarer,

- Classe $2: 43$ verbes intransitifs comme plaisanter ou fulminer,

- Classe 3 : 71 verbes transitifs, qui ne sont pas des verbes de discours rapporté, comme commenter, continuer ou interrompre dont le fonctionnement sera expliqué ci-dessous.

Ce résultat est surprenant à double titre : d'une part, seule la moitié des verbes sont des verbes de discours rapporté ; d'autre part, si les classes 1 et 2 sont fréquemment mentionnées dans la littérature, l'existence de la Classe 3 est généralement passée sous silence ${ }^{1}$.

Ainsi, Bonami \& Godard (2008) considèrent que la citation a toutes les caractéristiques d'un objet direct extrait $^{2}$, mais leur étude repose exclusivement sur les verbes de discours rapporté (c'est-à-dire des verbes de notre Classe 1) : elle ne fonctionne pas pour la moitié des verbes de citation que nous avons identifiés.

Lamiroy \& Charolles (2008) étudient les verbes de discours rapporté (Classe 1) et les intransitifs (Classe 2). Ils défendent l'hypothèse que les « verbes de parole » — classe de verbes qu'ils définissent sémantiquement 
— sont fondamentalement transitifs, avec un objet direct exprimant la parole émise. Ils sont amenés à définir une échelle de transitivité pour prendre en compte qu'un verbe comme plaisanter apparaît en incise de citation (6a), mais pas en discours rapporté (6b) : plaisanter est « moins » transitif que dire.

(6) a. “Je m'opposerai à cette loi”, plaisanta le sénateur.

b. *Le sénateur a plaisanté qu'il s'opposerait à cette loi.

Toutefois, attribuer un pseudo-objet direct à plaisanter au prétexte qu'il peut être un verbe de citation ne paraît pas linguistiquement justifié ${ }^{3}$. De plus, l'hypothèse de Lamiroy \& Charolles (2008) ne tient pas pour les verbes transitifs de la Classe 3 comme interrompre ou commenter, pour lesquels l'objet direct ne réfère pas à la parole émise, comme nous allons le montrer dans les sections suivantes, en analysant ces verbes en détail. Cette classe de verbes a été ignorée de la littérature, à l'exception de (Delaveau, 1988) (cf. ci-dessus). Elle se divise en deux sous-classes, la Classe 3A pour les verbes dont l'objet peut être animé, et la Classe 3I pour ceux dont l'objet est nécessairement inanimé. Comme nous allons le voir, le devenir de l'argument sémantique correspondant à cet objet direct au sein d'une DIC diffère selon la sous-classe.

Cependant, tous les verbes de la Classe 3 ont en commun que la citation correspond à un objet oblique dans la construction sans citation. L'étude successive des Classes 3A (Section 3.1) et 3I (Section 3.2) nous conduira à proposer une analyse discursive pour les DIC dont le verbe de citation est de la Classe 3 (Section 3.3), et à proposer pour ces verbes, lorsqu'ils sont employés comme verbes de citation, la définition de deux cadres de sous-catégorisation distincts, l'un de niveau syntaxico-phrastique et l'autre de niveau sémantico-discursif. Nous montrons ensuite que cette analyse discursive est également valable pour les verbes des Classes 1 (Section 3.4) et 2 (Section 3.5). Cette position est renforcée par le caractère poreux des frontières entre ces classes (Sections 3.6 et 3.7).

\subsection{Les verbes de la Classe $3 A$ (à objet animé)}

Considérons en premier lieu le verbe interrompre. Pour les emplois qui nous intéressent, ce verbe apparaît dans les exemples illustrés en (7).

Lorsqu'il n'est pas employé dans le contexte d'une citation, interrompre prend un objet direct obligatoire et un objet oblique facultatif introduit par la préposition par (ou éventuellement la préposition avec) (7a). L'objet direct correspond au propos qui est interrompu ou à qui les émet, par un processus de métonymie nous notons cet argument sémantique $X$ 一, et l'objet oblique décrit une propriété de l'interruption — nous notons $Y$ l'argument sémantique correspondant. Ces deux compléments sont nécessairement nominaux : les phrases (7b) et (7c) sont agrammaticales. Le verbe transitif interrompre n'est donc pas un verbe de discours rapporté.

(7) a. Le député a interrompu (le discours de) la Ministre (par une remarque acerbe).

b. *Le député a interrompu que les impôts allaient être réduits (par une remarque acerbe).

c. *Le député a interrompu (le discours de) la Ministre par (ce) qu'il trouvait cela anticipé.

Lorsque interrompre est employé comme verbe de citation, comme dans les exemples en (9), dont le premier est forgé et les autres attestés ${ }^{4}$, il garde le même sens. La citation est alors l'interruption elle-même et correspond ainsi à l'argument $Y$, qui ne peut donc pas être maintenu dans l'incise. Les exemples (9) montrent qu'une incise de citation peut comporter un objet direct, référant alors nécessairement à un animé ${ }^{5}$, sous forme de clitique ou de syntagme nominal — l'objet oblique étant quant à lui interdit. Ces exemples, nombreux, contredisent l'affirmation usuelle selon laquelle une incise de citation ne peut pas comporter d'objet 
direct. Ceci a pour conséquence que la citation ne peut en aucun cas être assimilé à un objet direct du verbe de citation, contrairement à ce que proposent par exemple Lamiroy \& Charolles (2008), Bonami \& Godard (2008) ou encore Riegel et al. (1994).

(9) a. "C'est complètement abscons", (l') interrompit le député.

b. - "Eh bien", l'interrompit-il, "c'est toujours la même crainte pour Maleborge?" [Gérard Hervé, Les hérésies imaginaires, p. 70]

c. - Mais, l'interrompis-je, comment lanciez-vous votre balle si droit (...) ? [Cyrano de Bergerac, L'autre monde ou les états et empires de la lune, p. 20]

d. - Tu sais, l'interrompis-je, je te ferai tout de même avoir ta Croix de guerre. [Blaise Cendrars, L'homme foudroyé, p. 360]

e. "A moins que", interrompit-il son ami d'une voix basse mais intelligible (...) [Google]

Autres verbes sur le modèle d'interrompre : couper, contredire, dégommer.

Tournons-nous vers le verbe apostropher. Contrairement à interrompre, dont l'objet peut être un animé par métonymie, apostropher se construit avec un objet direct obligatoirement humain, qui correspond à l'argument sémantique $X$ - la personne apostrophée —, et facultativement avec un objet oblique correspondant à l'argument sémantique $Y$ qui qualifie le propos utilisé pour apostropher (10a). Ici encore, ce verbe peut être la tête d'une incise de citation comportant généralement un objet direct (clitique ou syntagme nominal) (10b) et (11a-d). En réalité, les exemples comme (11e) où l'incise ne comporte pas d'objet direct semblent rares. L'objet oblique, quant à lui, est naturellement interdit dans la citation puisqu'il aurait le même rôle sémantique que la citation (*Et où allez-vous ainsi chargé ?" l'apostropha-t-il par une invective grossière).

(10) a. Le président du Parlement européen a apostrophé Vladimir Poutine (par une invective osée).

b. "La Russie est-elle encore une démocratie", l'a-t-il apostrophé en substance. [Google]

(11) a. "Salut, Jules", l'apostropha-t-il, lui barrant le chemin. [Jean Malaquais, Planète sans visa, p. 162]

b. - Et où allez-vous ainsi chargé ? l'apostropha-t-il. [Anna Gavalda, La consolante, p. 237]

c. "Comment", apostropha-t-il son ami, "tu oses donner asile à cette femme?" [Théodore Hersart de La Villemarqué, Barzaz Breiz, p. 30]

d. - Salaud, misérable, crapule! apostropha-t-il Dan Yack en secouant la table (...). [Blaise Cendrars, Dan Yack / Le plan de l'aiguille, p. 96]

e. - Maxime, apostropha-t-il durement, est-ce donc pour l'amour de Mademoiselle Clarice que vous portez des violettes? [Rosalia Colmeiro, La Couronne des Elfes, p. 175]

Autres verbes sur le modèle d'apostropher : aborder, accoster, héler, invectiver, narguer. On trouve ainsi des exemples tels que (12).

(12) a. - Asher di Simon da Modena ? héla-t-il. [Maud Tabachnik, Le sang de Venise, p. 88]

b. - Monsieur Perret, Monsieur Perret! le héla-t-elle. [Emanuelle Delle Piane, Voyage au pays des fées, p. 100]

c. - Hey, you bloody liar! le héla-t-elle en lui montrant son poing gauche [Anna Gavalda, La consolante, p. 359]

d. - Maria! héla-t-il sa vieille bonne. [Alphonse Allais, Euvres posthumes: Le journal, vol. 5, p. 308] 
Enfin, considérons le verbe menacer. Avec un sujet humain, il se construit avec un objet direct humain (non obligatoirement réalisé) — argument $X$ référant à qui est menacé — et un complément introduit par la préposition de qui peut être réalisé sous forme d'infinitive — argument $Y$ réferant à la menace — (13a). Lorsqu'il est employé comme verbe de citation, la citation correspond à $Y$. Cet argument ne peut donc pas être réalisé dans l'incise. Comme pour interrompre ou apostropher, $X$ peut apparaître comme clitique ou syntagme nominal dans l'incise (13c) et (14).

(13) a. Aimey Mbinah a menacé (Ali Bongo) de prendre le pouvoir.

b. "Si les autorités donnent Ali Bongo vainqueur et envoient les militaires, nous deviendrons les militaires et eux les civils. Le peuple vaincra et le candidat du PDG (Parti démocratique gabonais, au pouvoir, ayant investi Ali Bongo) quittera le Gabon”, menace Aimery Mbinah, forestier. [AFP]

c. "Le peuple vaincra", (le) menace Aimery Mbinah.

(14) a. "Malheur à toi si tu refuses, le menaça-t-il. Le Messie viendra et tu ne seras plus là pour l'accueillir." [Élie Wiesel, Silences et mémoire d'hommes, p. 48]

b. - Tu me paieras ça très cher, Auguste, le menaça-t-il. [Michel Verrier, La Taille de la SaintVincent, p. 396]

c. "tu vas regretter", l'a-t-il menacé. [Google]

d. - (...) Savez-vous que vous pointez une arme à un agent fédéral ? menaça-t -il son assaillant. [Google]

Autres verbes sur le modèle de menacer : accuser, convaincre.

Nous avons donc identifié trois séries de verbes dans la Classe 3A. Tous permettrent un objet direct dans l'incise de citation, pour peu qu'il soit animé. Ceci montre que la citation elle-même n'est pas un objet direct extrait.

Pour schématiser ces données, on peut poser que la structure argumentale d'un verbe de la Classe 3A employé hors contexte de citation, qui est donnée en (15a), est réalisée dans un contexte citationnel par la structure donnée en (15b). Nous notons $x$ l'argument sémantique correspondant au sujet (humain) du verbe.

(15) a. $\left[\mathrm{N}_{0+\text { hum }}\right]_{x} \quad \mathrm{~V}_{3 A}\left[\mathrm{~N}_{1}\right]_{X}$ Prép $\left[\mathrm{N}_{2}+\mathrm{V}_{\text {inf }}\right]_{Y}$

b. "[Citation $]_{Y}$ ", $\mathrm{V}_{3 A}\left[\mathrm{~N}_{0+\mathrm{hum}}\right]_{x}\left(\left[\mathrm{~N}_{1+\mathrm{hum}}\right]_{X}\right)$

\subsection{Les verbes de la Classe 3l (à objet inanimé)}

Les verbes de la Classe 3I sont des verbes transitifs dont l'objet ne peut être un animé. Le parangon de cette classe est le verbe commenter. Lorsqu'il n'est pas employé dans le contexte d'une citation, commenter prend un objet direct obligatoire et un objet oblique facultatif introduit par la préposition par (ou éventuellement la préposition avec) (16a). L'objet direct correspond à ce qui est commenté — argument sémantique noté $X$ - et l'objet oblique décrit une propriété du commentaire — l'argument sémantique correspondant au commentaire est noté $Y$. Ces deux compléments sont nécessairement nominaux : les phrases (16b) et (16c) sont agrammaticales. Le verbe transitif commenter n'est donc pas un verbe de discours rapporté.

(16) a. Le député a commenté cette décision (par une remarque acerbe). 
b. *Le député a commenté que les impôts allaient être réduits (par une remarque acerbe).

c. *Le député a commenté cette décision par (ce) qu'il trouvait cela anticipé.

Lorsque commenter est employé comme verbe de citation, comme par exemple en (17a), il garde le même sens mais le commentaire $Y$ est donné dans la citation, l'argument $X$ devant être récupéré dans le contexte gauche, par exemple en (17a), $X$ correspond à la décision (du gouvernement) de réduire les impôts (qui est l'antécédent du pronom neutre $c$ '). Contrairement à ce qui se passe pour les verbes étudiés à la section précédente, l'argument $X$ ne peut pas être réalisé dans l'incise (ni comme un GN *a commenté le député cette décision ni comme un clitique *l'a commentée le député). Cependant, cet argument ne peut pas être non spécifié : il doit nécessairement être retrouvé dans le contexte gauche de la citation, qui doit donc être non vide. Il est en effet impossible de commencer un discours par une citation introduite par commenter, même si la citation ne comporte aucun élément anaphorique, comme le montre l'incohérence du discours (17b) (incohérence notée par le signe \#).

(17) a. Le gouvernement a annoncé sa décision de réduire les impôts. "C'est prématuré", commenta le député.

b. [contexte nul] \#“Les paysans vont descendre dans la rue”, commenta le député.

En résumé, commenter a trois arguments sémantiques : $x$ le commentateur, $X$ ce qui est commenté, et $Y$ le commentaire. Quand il n'est pas employé comme verbe de citation, $x$ est réalisé en sujet, $X$ en objet direct et $Y$ est réalisé comme un complément oblique facultatif (ce qui veut dire que $Y$ peut être non spécifié). Ces données peuvent être enregistrées dans un cadre de sous-catégorisation standard, qui établit les contraintes syntaxiques et sémantiques et la correspondance entre les arguments syntaxiques et sémantiques (linking). Quand commenter est employé comme verbe de citation, seul $x$ est réalisé dans l'incise : $X$ doit être trouvé dans le contexte discursif gauche de la phrase comportant la citation et son incise, et $Y$ correspond à la citation.

Sur le même modèle que commenter, on peut citer les verbes suivants : continuer, nuancer, poursuivre, résumer.

Les structures argumentales correspondantes sont données en (18).

(18) a. $\left[\mathrm{N}_{0+\text { hum }}\right]_{x} \mathrm{~V}_{3 I}\left[\mathrm{~N}_{1-\text { hum }}\right]_{X}$ Prép $\left[\mathrm{N}_{2}\right]_{Y}$

b. $\cdots[\cdots]_{X} \cdots$. "[Citation $]_{Y}$ ", $\mathrm{V}_{3 I}\left[\mathrm{~N}_{0+\text { hum }}\right]_{x}$

\subsection{Analyse discursive pour les verbes de la Classe 3}

À ce stade, la question de la nature de la relation entre citation et verbe de citation se pose en les termes suivants. Premièrement, pour tous les verbes de la Classe 3, la citation correspond à l'argument $Y$, qui est un objet oblique dans la construction de base (et pas un objet direct). Deuxièmement, dans un DIC, la citation n'est pas un objet direct extrait, comme nous l'avons vu en étudiant les verbes de la Classe 3A — le véritable objet direct, lorsqu'il réfère à un humain, peut apparaître dans l'incise. Troisièmement, lorsqu'un verbe de la Classe 3I est employé dans une incise de citation, son argument $X$ (qui est objet direct dans la construction de base, et qui correspond à ce qui est commenté, continué, nuancé, etc.) est nécessairement à retrouver par un procédé de niveau discursif (dans le contexte discursif gauche).

Sauf à introduire une fonction syntaxique ad hoc pour les citations, l'analyse des verbes de la Classe 3 conduit ainsi à postuler que la citation elle-même, qui correspond à l'argument sémantique $Y$, est elle aussi 
à relier par un procédé de niveau discursif au verbe de citation. Il n'y a donc pas de relation argumentale de niveau syntaxique entre la citation et le verbe de citation. La citation correspond sémantiquement (mais non syntaxiquement) au second objet, s'il existe. Cependant, qu'un verbe soit employé dans un contexte de base (sans citation) ou dans un DIC en tant que verbe de citation, son sens ne change pas. Nous posons donc pour ces verbes l'existence d'une redistribution qui construit la structure argumentale pour les verbes de citation (de la Classe 3) à partir de leur structure argumentale de base, redistribution qui fait disparaître du cadre syntaxique l'argument sémantique $Y$ (et éventuellement l'argument sémantique $X$ lorsqu'il ne peut être réalisé). Cette modélisation par une redistribution, qui sera généralisée aux verbes des Classes 1 et 2 , sera présentée plus en détails à la section 4.

Cette analyse discursive revient à dire que la citation et l'incise forment un segment de discours composé de deux blocs, qui ne peuvent apparaître l'un sans l'autre. La syntaxe n'intervient que pour contraindre la position respective de ces deux blocs (voir Section 2), pour imposer l'inversion du sujet dans l'incise, et pour établir les arguments qui peuvent être réalisés dans l'incise en précisant leur forme (voir Section 4). Il n'existe aucun lien argumental de niveau syntaxique entre ces deux blocs, en particulier aucune fonction syntaxique (ou typage de dépendance) entre le verbe de citation et la citation. Le seul lien qui existe entre ces deux blocs relève du niveau sémantico-discursif, qui permet aussi d'établir un éventuel lien entre la citation et le contexte gauche du segment de discours citation et incise (voir Section 5).

Ces données ne peuvent toutes pas être enregistrées dans un cadre de sous-catégorisation syntaxique standard, qui ne peut établir que des contraintes relevant du domaine phrastique. Il faut donc faire appel à deux cadres de sous-catégorisation :

- un au niveau phrastique qui établit principalement les contraintes syntaxiques,

- un au niveau discursif qui établit principalement les contraintes sémantiques.

Postuler l'existence de deux cadres de sous-catégorisation, l'un au niveau phrastique, l'autre au niveau discursif, peut sembler un peu "hérétique". Néanmoins, rappelons que les connecteurs de discours adverbiaux comme ensuite ou de ce fait demandent deux cadres de sous-catégorisation. En effet, au niveau syntaxique, ces adverbiaux n'ont qu'un seul argument ${ }^{6}$. En revanche, au niveau sémantique, ces connecteurs ont deux arguments, dont l'un doit être retrouvé dans le contexte discursif gauche de la phrase où le connecteur apparaît. Cette différence entre le nombre d'arguments syntaxiques et sémantiques ne peut être prise en compte qu'en faisant appel à deux cadres de sous-catégorisation : l'un postulant les contraintes syntaxiques observées au niveau phrastique, l'autre postulant les contraintes sémantiques observées au niveau discursif.

Postuler l'existence de ces deux cadres de sous-catégorisation demande de mettre au point une interface syntaxe-sémantique non triviale, qui repose sur une interface phrase-discours. Il n'est pas dans le champ de cet article de discuter d'une telle interface ${ }^{7}$. Néanmoins, en ce qui concerne les verbes qui peuvent être la tête d'une incise de citation, nous présentons à la Section 4 une ébauche de leur cadre de sous-catégorisation phrastique et à la Section 5 une ébauche de leur cadre de sous-catégorisation discursif.

Ayant posé une analyse discursive pour les DIC dont le verbe de citation est de la Classe 3, examinons la validité de cette analyse pour les autres verbes de citation.

\subsection{Analyse discursive pour les verbes de la Classe 1}

Pour les verbes transitifs de la Classe 1 - qui permettent un objet direct de forme Que $P$-, on peut envisager deux solutions pour analyser le lien entre la citation et (la tête de) l'incise de la citation : 
- analyse syntaxique : la citation est liée syntaxiquement à l'incise, c'est un objet extrait comme argumenté par Bonami \& Godard (2008);

- analyse discursive : la citation et son incise forment un segment de discours, sans lien de dépendance syntaxique, comme c'est le cas pour les incises dont le verbe est de la Classe 3.

Nous optons pour l'analyse discursive pour les raisons suivantes ${ }^{8}$.

D'abord, elle nous permet de rendre compte du fait qu'un DIC ne peut pas être enchâssé, comme signalé dans les exemples (5), répétés ci-dessous en (19).

(19) a. *Je crois que "donne-moi la main !" a lancé Paul à Marie. [(Bonami \& Godard, 2008)]

b. *Je suis furieuse parce que "Tu es paresseuse", m'a dit Fred.

En effet, le phénomène d'extraction ne peut pas justifier à lui seul l'interdiction d'enchâssement, comme en témoigne l'acceptabilité des exemples en (20a-b) où l'objet est extrait dans une clivée qui est enchâssée. A rebours, la formation d'un segment de discours - par exemple, deux clauses reliées par un connecteur de discours (de ce fait) - élimine toute possibilité d'enchâssement, comme en témoigne l'inacceptabilité des exemples en (20c-d).

(20) a. Je crois que c'est Fred que Sue aime.

b. Je suis furieuse parce que c'est Fred que Sue aime.

c. *Je crois que [Sue aime Fred, de ce fait elle est heureuse].

d. *Je suis furieuse parce que [Sue aime Fred, de ce fait elle est heureuse].

L'analyse syntaxique n'explique pas non plus pourquoi la citation ne peut pas se trouver sous la portée d'une négation, sauf pour un verbe comme cacher ou taire, voir (3). En effet, de tels phénomènes d'extraction ne sont en rien bloqués par la négation (C'est Fred que Sue n'aime pas).

Ensuite, l'analyse par extraction n'explique pas pourquoi l'incise ne peut apparaittre avant le citation que dans les cas où elle est précédée d'un connecteur, voir (4b) de la Section 2. Une analyse discursive semble plus adéquate pour rendre compte de cette contrainte.

L'analyse discursive est aussi plus adéquate pour rendre compte des données suivantes : certains verbes de discours rapporté tels que ajouter ou répondre ne peuvent pas être la tête d'une incise de citation dans un DIC qui figure dans un contexte gauche nul : (21a) est incohérent (dans un contexte nul) contrairement à (21b).

(21) a. [contexte nul] \#"Les paysans vont descendre dans la rue", répondit le député.

b. Le Premier ministre a demandé comment les paysans allaient réagir à cette loi. "Les paysans vont descendre dans la rue", répondit le député.

Enfin, l'analyse discursive permet de proposer une solution homogène pour tous les DIC, quel que soit le verbe de citation. En effet, l'analyse discursive s'impose aussi pour les verbes intransitifs de la Classe 2, comme nous allons le montrer.

\subsection{Analyse discursive pour les verbes de la Classe 2}

Tout d'abord, à l'instar des connecteurs de discours (Section 3.2), ces verbes de citation ont un argument de plus au niveau sémantico-discursif par rapport au niveau syntaxico-phrastique. Par exemple, pour le verbe 
intervenir, l'argument sémantique $Y$ correspondant à l'intervention de l'intervenant est réalisé en (23b) il correspond à la citation — mais il ne peut pas être réalisé que dans une citation : il n'a pas d'équivalent syntaxique en $(23 a)^{9}$. Cette différence entre le nombre d'arguments syntaxiques et sémantiques ne peut être prise en compte qu'en faisant appel à deux cadres de sous-catégorisation, l'un syntaxico-phrastique, l'autre sémantico-sémantique.

(23) a. Le député est intervenu dans la discussion.

b. "C'est abscons", est intervenu le député (dans la discussion).

Ensuite, la plupart des verbes de la Classe 2 imposent que le DIC figure dans un contexte gauche non nul, comme le montre l'incohérence des discours (24) énoncés dans un contexte gauche nul. Rappelons que cette contrainte peut facilement être prise en compte dans une analyse discursive.

(24) a. [contexte nul] \#“Les paysans vont descendre dans la rue”, intervint le député.

b. [contexte nul] \#“Les paysans vont descendre dans la rue”, plaisanta le député.

En conclusion, nous proposons que tous les DIC — quelle que soit la classe du verbe de citation - soient considérés comme des segments de discours constitués de deux blocs sans lien syntaxique. Cette analyse discursive demande deux cadres de sous-catégorisation pour les verbes de citation, qui seront présentés respectivement dans les sections 4 et 5 . Auparavant, nous allons discuter des frontières entre les Classes 1 et 3 d'une part, et 1 et 2 d'autre part.

\subsection{Frontière entre les Classes 1 et 2}

Considérons les verbes exprimant des cris d'animaux. Gross (1975) les a tous classés dans la Table 9 (NO V que P à N2) avec un signe + dans la colonne indiquant la possibilité d'être employé comme verbe de citation, ce qui revient à les classer dans notre Classe 1 , et donc à considérer comme acceptables les exemples en (25).

(25) a. Luc a miaulé à sa chérie qu'il l'aimait.

b. "Je t'aime", a miaulé Luc à sa chérie.

Dans leur étude qui repose sur un corpus d'essais écrits entre 1950 et 2000 issu de Frantext, Lamiroy \& Charolles (2008) ont noté 35 occurrences de glapir employé comme verbe de citation, cinq de croasser et zéro de coasser. Ils indiquent deux occurrences de glapir comme verbe de discours rapporté et apparemment zéro pour croasser et coasser ${ }^{10}$.

Dans notre corpus de dépêches de l'AFP, seul le verbe aboyer figure comme verbe de citation exprimant un cri d'animal et il est de la Classe 1.

Ces données montrent d'une part qu'il est difficile de dresser la liste des verbes de citation dans la mesure où cette liste est mouvante selon le genre de textes (littéraire, journalistique, etc.). D'autre part, elles montrent que la frontière entre les Classes 1 et 2 est aussi mouvante. Il est donc particulièrement important de proposer la même analyse des verbes de citation, que ceux-ci soient de la Classe 1 ou 2, et c'est ce que permet de faire l'analyse discursive proposée ici. 


\subsection{Frontière entre les Classes 1 et 3}

Certains verbes sont classés dans la Classe 3, bien que leur objet direct puisse être une complétive. C'est le cas pour expliquer pour lequel nous considérons qu'il a deux entrées, une dans la Classe 3, l'autre dans la Classe 1 (il a aussi deux entrées dans la Table 9 de Gross (1975)). Dans les exemples (27), il est employé dans un sens proche de justifier, motiver. C'est un verbe de la Classe 3 qui permet que l'argument $X-$ ce qui est expliqué — soit une complétive $(27 b)^{11}$.

(27) a. Le député a expliqué la hausse du prix du riz par des spéculations outrancières.

b. Le député a expliqué que le riz subissait une hausse spectaculaire par des spéculations outrancières.

c. Le prix du riz subit une hausse spectaculaire. "Il y a eu des spéculations outrancières", a expliqué le député.

Dans les exemples (28), expliquer est employé dans un sens proche de enseigner, faire comprendre, sans qu'aucune notion de causalité ne soit en jeu. C'est un verbe de la Classe 1.

(28) a. Le professeur a expliqué qu'un triangle isocèle avait par définition deux côtés de longeurs égales.

b. "Un triangle isocèle a par définition deux côtés de longeurs égales", a expliqué le professeur.

Pour d'autres verbes, on peut hésiter entre deux entrées ou une seule. C'est le cas de préciser. Dans le paradigme (29), c'est apparemment un verbe de la Classe 3, qui permet que l'objet direct soit une complétive (29b). (29a) peut être paraphrasé par «Une déclaration fracassante du chercheur a rendu sa pensée plus précise ».

(29) a. Le chercheur a précisé sa pensée par une déclaration fracassante.

b. Luc nous a annoncé qu'il arriverait demain. Il a précisé qu'il arriverait à 14 h pétantes.

c. Luc nous a annoncé qu'il arriverait demain. “J'arriverai à 14h pétante”, a-t-il précisé.

Dans le paradigme (30), préciser peut être paraphrasé par indiquer de façon précise, et c'est apparemment un verbe de la Classe 1.

(30) a. Le Ministre du budget a précisé la position du gouvernement sur la TVA.

b. Le Ministre du budget a précisé qu'il n'était pas question d'augmenter la TVA.

c. "Il n'est pas question d'augmenter la TVA", a précisé le Ministre du budget.

Néanmoins, il n'est pas clair que l'on ait affaire à deux entrées distinctes de préciser, ce qui milite en faveur d'une analyse commune pour les DIC dont le verbe de citation est de la Classe 1 ou 3.

\section{Lexique syntaxique des verbes de citation}

Nous avons intégré les données lexicales rassemblées au cours de notre étude de corpus, analysées au regard des résultats présentées jusqu'ici, au sein du lexique Lefff(Lexique des formes fléchies du français) (Sagot, 2010). Le Lefffest un lexique librement disponible à large couverture, qui repose sur le formalisme lexical Alexina. Outre les informations de niveau morphologique, il associe des informations syntaxiques à 
chaque entrée « intensionnelle » d'un lemme donné, chacune correspondant à un sens différent du lemme. Ces informations syntaxiques comprennent, entre autres, un cadre de sous-catégorisation initial (fonctions syntaxiques et leurs possibles réalisations) et les redistributions acceptables, qui produisent automatiqument différents cadres de sous-catégorisation finaux (pour les verbes par exemple, les redistributions possibles sont \%actif, \% passif, \%impersonnel, \%se_moyen, etc.). Le modèle lexical Alexina est indépendant de tout formalisme grammatical, ce qui permet aux lexiques Alexina d'être utilisés par des systèmes d'analyse syntaxique reposant sur divers formalismes grammaticaux (TAG, LFG ...).

Lorsqu'un verbe est la tête d'une incise de citation, il a le même sens que lorsqu'il est utilisé dans un contexte sans citation. Par exemple, rappelons que commenter a le même sens dans (16a) et dans (17a). De plus, comme souligné par Bonami \& Godard (2008), le verbe de citation impose des restrictions de sélection sur le type d'acte de parole que la citation peut exprimer, et ces restrictions sont cohérentes avec celles observées dans les contextes sans citation. Par exemple, le verbe demander, lorsqu'il est utilisé en tant que verbe de citation, impose à la citation d'être une interrogative (31a), en cohérence avec son emploi de base avec une interrogative indirecte (31b). À l'inverse, affirmer ne se construit qu'avec des assertions (31c-d).

(31) a. "Quelle heure est-il ?", demanda-t-il.

b. Il demanda quelle heure il était.

c. "Il est 2h30", affirma-t-il.

d. Il affirma qu'il était $2 \mathrm{~h} 30$.

Nous modélisons donc la possibilité pour un verbe d'être la tête d'une incise de citation dans un DIC en ajoutant à son entrée lexicale une nouvelle redistribution. Cette redistribution, préfixée par \%DIC, est définie comme suit :

- elle élimine toutes les fonctions syntaxiques qui ne peuvent pas être réalisées dans une incise de citation, à savoir : (i) dans tous les cas, l'argument qui correspond à la citation elle-même (ce qui est sans effet pour les verbes de la Classe 2); (ii) pour certains verbes de la Classe 3I, l'objet direct (Obj), comme expliqué à la section 3.2. Ceci induit plusieurs redistributions \%DIC, selon la classe du verbe et la fonction syntaxique correspondant à la citation; par exemple, \%DIC_noObj_noObl, associé aux verbes de la Classe 3I tels que commenter, élimine l'Obl, qui correspond à la citation, ainsi que l'Obj;

- elle élimine toute réalisation phrastique (scompl) ou infinitive (sinf) du sujet (Suj), ce dernier devant être un animé;

- elle ajoute un trait spécifique [DIC=+], destiné être utilisé par la grammaire pour forcer l'inversion du sujet et l'identification d'une incise de citation;

- elle ajoute un trait qui représente les restrictions de sélection qui affectent la citation, tel que [assertion=+] ou [interrogative=+]; ceci permet à la sortie d'un analyseur syntaxique d'être compatible avec une analyse discursive ultérieure.

Par exemple, des entrées (simplifiées) du Lefffpour les verbes dire (Classe 1), ricaner (Classe 2) et commenter (Classe 3) sont montrées respectivement en (32a), (32c) et (32e). Chacune de ces entrées « intensionnelles » produit différentes entrées « extensionnelles », pour chaque combinaison d'une forme fléchie et d'une redistribution compatible. Pour chacun de ces trois verbes, nous indiquons l'entrée extensionnelle (simplifiée) pour la redistribution DIC et la troisième personne du singulier du passé simple, respectivement en (32b), (32d) et (32f). Par exemple, la redistribution \%DIC_noObj produit une entrée pour dit dans laquelle l'Obj est éliminé, mais l'Objà optionnel est conservé, puisqu'il peut être réalisé dans l'incise de citation.

(32) a. dire Suj:cln|sn,

Obj:cla|sn|de-sinf|scompl|qcompl, 


\author{
Objà:(cld|à-sn);; \\ $\%$ actif,\%passif,\%se_moyen, \\ \%passif_impersonnel,\%DIC_noObj \\ b. dit Suj:cln|sn,Objà:(cld|à-sn);DIC=+,assertion=+; \\ c. ricaner Suj:cln|sn;;\%active,\%DIC_intrans \\ d. ricana Suj:cln|sn;DIC=+, assertion=+; \\ e. commenter Suj:cln|sn,Obj:cla|sn, \\ Obl:(par-sn);; \\ $\%$ actif,\%passif,\%se_moyen, \\ $\% D I C \_n o O b j \_n o O b l$ \\ f. commenta Suj:cln|sn;DIC=+;assertion=+
}

\title{
5 Lexique discursif pour les verbes de citation
}

Nous allons présenter les cadres de sous-catégorisation sémantico-discursifs pour certains verbes de citation en fonction de leur classe syntaxique, avant de dresser un bilan sur ces cadres.

\subsection{Verbes de la Classe 1}

Pour le verbe prototypique dire employé comme verbe de citation, le cadre de sous catégorisation sémantico-discursif est donné (sous forme simplifiée) en (33). Il indique que dire a trois arguments sémantiques : $x$ et $y$ qui sont des entités humaines correspondant respectivement au sujet et à-obj de dire, $Y$ qui correspond au segment de discours formé par la citation. L'incise de citation est représentée en $W$. Ce segment de discours est centré autour de l'événement $e$ représentant le dire de $x$ à $y$ (si l'incise de citation comporte des ajouts (dit-il à Marie après le dîner), leur représentation sémantique est incluse dans $W$ ). Les segments $W$ et $Y$ sont reliés par la relation de discours Attribution, qui a été proposée dans la littérature anglophone pour relier un segment attributif à son segment attribué, (Carlson et al., 2003 ; Wolf \& Gibson, 2006 ; Redeker \& Egg, 2006 ; Hunter et al., 2006) $)^{12}$.

(33) $\lambda x y Y . \exists W(W \cong(\exists e \cdot \operatorname{dire}(\mathrm{e}, \mathrm{x}, \mathrm{y})) \wedge \operatorname{Attribution}(W, Y))$ avec $x, y:: e, Y, W:: t$

Nous posons que Attribution impose la contrainte suivante sur son premier argument, le segment attributif noté ici $W$ : $W$ doit poser l'existence d'un événement $e$ qui doit être typé comme acteParole, le concept prototypique. C'est le cas pour le cadre de sous-catégorisation de dire présenté en (33) puisqu'on a dire $(\mathrm{e}, \mathrm{x}) \rightarrow$ acteParole $(\mathrm{e}, \mathrm{x})$. Cette contrainte exclut que $W$ soit défini comme la négation d'un événement. Elle rend donc compte du fait qu'une incise de négation ne peut pas être sous la portée d'une négation, sauf lorsque sa tête est un verbe comme cacher qui doit obligatoirement être nié, voir (3). On peut poser l'existence d'une entrée verbale ne_pas_cacher qui correspond à un sous-type de acteParole, avec ne_pas_cacher $(\mathrm{e}, \mathrm{x}) \rightarrow$ acteParole $(\mathrm{e}, \mathrm{x})$.

Pour le verbe annoncer, son cadre de sous-catégorisation sémantico-discursif est celui présenté en (33) en remplaçant dire $(e, x, y)$ par annoncer $(e, x, y)$. On peut en effet considérer que annoncer est une spécification de acteParole. Par contre, pour répondre, qui demande que le DIC apparaisse dans un contexte gauche non 
vide, voir (21) à la Section 3.4, nous proposons le cadre de sous catégorisation en (34a). Il indique que le verbe de citation répondre a trois arguments : $x$ qui est une entité humaine correspondant à son sujet, $Y$ qui correspond à la citation, et $X$ qui est un segment de discours qui doit être trouvé dans le contexte gauche du DIC. $Y$ et $X$ sont reliés par la relation de discours Réponse proposée par Asher \& Lascarides (2003). Dans (34a), le segment attributif $W$ n'est pas une représentation fidèle de l'incise de citation : l'événement $e$ n'est pas typé par répondre, il est typé par acteParole ${ }^{13}$. Le cadre de sous-catégorisation en (34a) formalise le fait que le discours en (34b) peut être paraphrasé par (34c) où le verbe de citation est dire.

(34) a. $\lambda x Y X . \exists W(W \cong(\exists e \cdot \operatorname{acteParole}(\mathrm{e}, \mathrm{x})) \wedge \operatorname{Attribution}(W, Y) \wedge \operatorname{Réponse}(Y, X))$ avec $x:: e, X, Y, W:: t$

b. Le Premier ministre a demandé comment les paysans allaient réagir à cette loi. "Les paysans vont descendre dans la rue", répondit le député.

c. Le Premier ministre a demandé comment les paysans allaient réagir à cette loi. "Les paysans vont descendre dans la rue", dit le député en réponse à la question du Premier ministre.

Pour le verbe ajouter, son cadre de sous-catégorisation sémantico-discursif est celui de (34a) en remplaçant la relation de discours Réponse par Continuation introduite par Asher \& Lascarides (2003).

\subsection{Verbes de la Classe 2}

Pour un verbe intransitif de la Classe 2 tel que ricaner, le cadre de sous-catégorisation est donné en (35). Il indique que le verbe de citation ricaner a deux arguments, $x$ qui correspond au sujet du verbe et $Y$ à la citation. L'incise de citation est représentée au moyen de $W$ et $W_{1}: W$ représente seulement le rôle de segment attributif de l'incise, $W_{1}$ représente le fait que $x$ ricanait. Ces deux segments sont reliés par la relation ArrièrePlan, qui spécifie que $e$ a eu lieu pendant que $e_{1}$ se déroulait. Ce cadre de sous-catégorisation formalise l'analyse par fusion proposée par Gross (1975) : ricaner employé comme verbe de citation est équivalent à « dire en ricanant ».

(35) $\lambda x Y . \exists W W_{1}\left(W \cong(\exists e \cdot \operatorname{acteParole}(\mathrm{e}, \mathrm{x})) \wedge W_{1} \cong\left(\exists e_{1} \cdot \operatorname{ricaner}\left(\mathrm{e}_{1}, \mathrm{x}\right)\right) \wedge \operatorname{ArrièrePlan}\left(W, W_{1}\right) \wedge\right.$ $\operatorname{Attribution}(W, Y))$

Si l'on considère qu'un DIC dont le verbe de citation est ricaner ne peut pas être énoncé dans un contexte discursif gauche nul, voir (24b), alors il faut ajouter un troisième argument $X$ dans le cadre de sous-catégorisation de ce verbe (qui doit être trouvé dans le contexte discursif gauche), avec la relation Commentaire $(Y, X)$ (cf ci-dessous). Ceci revient à affiner l'analyse par fusion de Gross (1975) : ricaner employé comme verbe de citation est équivalent à « commenter en ricanant ».

\subsection{Verbes de la Classe 3}

Pour le verbe transitif commenter de la Classe 3I, le cadre de sous-catégorisation est donné en (36). Il indique que le verbe de citation commenter a trois arguments, $x$ qui correspond au sujet du verbe, $Y$ à la citation, et $X$, segment de discours qui doit être retrouvé dans le contexte discursif gauche du DIC. $Y$ est relié à $X$ par la relation de discours Commentaire, proposée par Danlos (2007).

(36) $\lambda x Y X . \exists W(W \cong(\exists e . \operatorname{ActeParole}(\mathrm{e}, \mathrm{x})) \wedge \operatorname{Attribution}(W, Y) \wedge \operatorname{Commentaire}(Y, X))$ 
Pour continuer, également de la Classe 3I, le cadre de sous-catégorisation est identique à (36) en remplaçant la relation Commentaire par Continuation. De façon similaire, pour expliquer, c'est la relation Explication qui est utilisée. Considérons le verbe éluder, également de la Classe 3I, dont l'argument X (ce qui est éludé) doit dénoter une question (37). Son cadre de sous-catégorisation sémantico-discursif est identique à celui de commenter, voir (36), en remplaçant Commentaire par la relation Réponse : le résultat est donc identique à (34a), le cadre de sous-catégorisation de répondre, de la Classe 1. Ceci montre que deux verbes n'appartenant pas à la même classe syntaxique peuvent avoir le même cadre de souscatégorisation sémantico-discursif. Néanmoins, il n'est pas entièrement satisfaisant de donner à répondre et éluder le même cadre car, pour éluder, l'information que $Y$ est une réponse élusive à la question $X$ est perdue. Il faut donc mettre au point un mécanisme permettant de récupérer cette information.

(37) a. L'équipe initiatrice de ce projet élude toute question sur ce sujet par une pirouette.

b. Parmi les événements qui ont marqué le centenaire de la naissance de Burle Marx, l'exposition "Roberto Burle Marx 100 ans : la permanence de l'instable”, a connu un grand succès à Rio. Depuis le 17 juillet, elle est installée à Sao Paulo avant une probable arrivée en Europe. En Allemagne? En France? “C'est un secret”, élude Isabela Ono de l'équipe initiatrice du projet. [AFP]

Pour un verbe de la Classe 3A comme le verbe héler, dont les emplois sont illustrés en (12), nous proposons le cadre de sous-catégorisation en (38). Ce verbe de citation a trois arguments, $x$ et $y$ qui sont les entités animées correspondant respectivement au sujet et à l'objet direct du verbe, et $Y$ qui correspond à la citation. L'incise de citation est représentée au moyen de la relation ArrièrePlan qui relie $W$ représentant l'aspect segment attributif de l'incise et $W_{1}$ qui représente le « hélage » de $y$ par $x$.

(38) $\lambda x y Y . \quad \exists W W_{1}\left(W \cong(\exists\right.$ e.acteParole $(e, x)) \wedge W_{1} \cong\left(\exists e_{1} \cdot\right.$ héler $\left.\left(e_{1}, x, y\right)\right) \wedge$ $\left.\operatorname{ArrièrePlan}\left(W, W_{1}\right) \wedge \operatorname{Attribution}(W, Y)\right)$

\subsection{Bilan}

Les cadres de sous-catégorisation sémantico-discursifs que nous avons présentés pour divers verbes de citation des trois classes comportent tous la forme logique $\operatorname{Attribution}(W, Y)$ où $Y$ correspond à la citation et $W$ est un segment de discours qui représente fidèlement ou partiellement l'incise de citation. $W$ représente fidèlement l'incise de citation quand le verbe de celle-ci désigne clairement un acte de parole, par exemple dire $(e, x) \rightarrow$ acteParole $(e, x)$. Lorsque le verbe de citation n'est que secondairement un verbe de parole, comme répondre, ou n'est pas du tout un verbe de parole, comme ricaner, alors $W \cong(\exists e$.acteParole $(\mathrm{e}, \mathrm{x}))$, c'est-à-dire que $W$ ne représente que l'aspect segment attributif de l'incise de citation (avec ses éventuels ajouts s'ils existent). Les informations spécifiques qui viennent du verbe de citation ont été représentées de l'une des deux façons suivantes :

- soit W est lié par la relation ArrièrePlan à un segment $W_{1}$ qui représente les informations spécifiques venant du verbe de citation ; c'est le cas pour ricaner de la Classe 2, voir (35), et pour héler de la Classe 3, voir (38);

- soit Y est lié par une relation de discours $R$ - qui dépend du verbe de citation — à un segment de discours $X$ qui figure dans le contexte gauche du DIC; c'est le cas pour répondre de la Classe 1, voir (35), et pour éluder de la Classe 3, avec $R=$ Réponse

La première solution semble ne pas poser de problème. Signalons qu'elle peut s'appliquer aux nombreux verbes de la Classe 1 qui spécifient une attitude de leur sujet, par exemple craindre, souhaiter ou réclamer. 
Ainsi pour réclamer, dont les emplois sont illustrés en (40a-b), nous proposons pour le verbe de citation le cadre de sous-catégorisation donné en $(40 \mathrm{c})$, où le symbole $\mathrm{g}(\mathrm{Y})$ représente le contenu de $Y^{14}$.

(40) a. Le député a réclamé que le gouvernement démissionne.

b. "Le gouvernement doit démissionner", a réclamé le député.

c. $\lambda x Y . \quad \exists W W_{1}\left(W \cong(\exists e \cdot \operatorname{acteParole}(\mathrm{e}, \mathrm{x})) \wedge W_{1} \cong\left(\exists e_{1}\right.\right.$.réclamer $\left.\left(\mathrm{e}_{1}, \mathrm{x}, \mathrm{g}(\mathrm{Y})\right)\right) \wedge$ $\left.\operatorname{ArrièrePlan}\left(W, W_{1}\right) \wedge \operatorname{Attribution}(W, Y)\right)$

Par contre, on rencontre des difficultés avec la seconde solution, difficultés déjà illustrées pour éluder : la formule Réponse $(Y, X)$ ne permet pas de représenter que $Y$ est une réponse élusive à $X$. D'une manière plus générale, la richesse d'expression des nombreux verbes de citation se laisse difficilement refléter dans la liste des relations de discours qui se limite grosso modo à une trentaine d'éléments.

Ajoutons qu'aucune des deux solutions présentées ne semble adéquate pour un verbe de citation comme menacer — dont les emplois ont été illustrés en (13) — si on considère que ce verbe n'est que secondairement un verbe de parole, autrement dit si on considère que $W$ n'est pas $\exists e$.menacer $(\mathrm{e}, \mathrm{x}, \mathrm{y})$. En considérant que $W \cong(\exists e$.ActeParole $(\mathrm{e}, \mathrm{x}, \mathrm{y}))$, alors il faut trouver une solution pour représenter le fait que le contenu de $Y$ (ou une partie de ce contenu) est une menace pour $y$.

Les entrées lexicales de niveau discursif que nous avons proposées dans cette partie sont donc certainement à affiner. Toutefois, le principe général du double cadre de sous-catégorisation reste convaincant, et semble de nature à modéliser de façon satisfaisante les données rassemblées sur les incises de citations et les verbes qui en sont la tête.

\section{Conclusion}

Nous avons présenté une analyse complète des discours constitués d'une citation et d'une incise de citation, dont la tête est un verbe dit verbe de citation. Nous avons mené à bien cette étude grâce à un corpus de dépêches de l'AFP, dans lequel ce type de constructions est particulièrement fréquent. Nous avons identifié trois classes de verbes de citation : des verbes transitifs de discours rapporté (dire, déclarer), des verbes intransitifs (plaisanter, fulminer) et des verbes transitifs qui ne sont pas des verbes de discours rapporté (interrompre, commenter, continuer), ces derniers étant souvent ignorés. Nous avons montré que la relation entre citation et incise de citation n'est pas purement phrastique, et notamment que la relation sémantique entre les deux ne peut être restituée qu'au niveau discursif, en prenant en compte le contexte discursif gauche de la phrase concernée.

Ces résultats nous ont conduit à proposer une modélisation lexicale des verbes de citation au sein du lexique syntaxique Lefff, afin de permettre la prise en compte de ces constructions dans les analyseurs syntaxiques automatiques. Ils nous ont également amené à proposer une analyse discursive détaillée de la relation entre citation et incise de citation, où le verbe de citation est associé à un cadre de sous-catégorisation discursif distinct de son cadre de sous-catégorisation phrastique. Il s'agit d'une première étape vers une prise en compte satisfaisante de ces constructions dans un analyseur syntaxique automatique, et de son extension vers le niveau discursif.

\section{Remerciements}

Nous remercions Olivier Bonami pour ses commentaires fructueux. 


\section{Références bibliographiques}

Asher, N. \& Lascarides, A. (2003). Logics of Conversation. Cambridge: Cambridge University Press.

Bonami, O. \& Godard, D. (2007). Quelle syntaxe, incidemment, pour les adverbes incidents? Bulletin de la Société de Linguistique de Paris, CII, 255-284.

Bonami, O. \& Godard, D. (2008). Syntaxe des incises de citation. In Actes du Premier Colloque Mondial de Linguistique Française, p. 209-225, Paris, France.

Carlson, L., Marcu, D. \& Okurowski, M. E. (2003). Building a discourse-tagged corpus in the framework of rhetorical structure theory. In J. van Kuppevelt \& R. Smith, Eds., Current Directions in Discourse and Dialogue, p. 85-112. Kluwer Academic Publishers.

Danlos, L. (2007). Integrating discourse relations into lexical semantics. In Proceedings of GL'07, Paris, France.

Danlos, L. (2009). D-STAG: a formalism for discourse analysis based on SDRT and using synchronous TAG. In Proceedings of the 14th Conference on Formal Grammar (FG'09), p. 1-20, Bordeaux, France.

de Cornulier, B. (1978). L'incise, la classe des verbes parenthétiques et le signe mimique. Cahier de linguistique, 8, 53-95.

Delaveau, A. (1988). La voix et les bruits : note sur les verbes introducteurs de discours rapporté. Revue LINX, 18, $125-136$.

Gross, M. (1975). Méthodes en syntaxe. Paris: Hermann.

Hunter, J., Asher, N., Reese, B. \& Denis, P. (2006). Evidentiality and intensionality: Two uses of reportative constructions in discourse. In Proceedings of the Constraints in Discourse Workshop (CID'06), Maynoth, Ireland.

Lamiroy, B. \& Charolles, M. (2008). Les verbes de parole et la question de 1?(in)transitivité. Revue Discours, 2.

Maier, E. (2009). Quotation and the semantics of speech report. In Proceedings of ESSLLI'09, Bordeaux, France.

Redeker, G. \& Egg, M. (2006). Says who? on the treatment of speech attributions in discourse structure. In Proceedings of the Constraints in Discourse Workshop (CID'06), Maynoth, Ireland.

Riegel, M., Pellat, J.-C. \& Rioul, R. (1994). Grammaire méthodique du français. PUF.

Sagot, B. (2010). The Lefff, a freely available, accurate and large-coverage lexicon for french. In Proceedings of LREC 2010, La Valette, Malte.

Sagot, B., Danlos, L. \& Stern, R. (2010). A lexicon of french quotation verbs for automatic quotation extraction. In Proceedings of LREC 2010, La Valette, Malte.

Webber, B. (2004). D-LTAG: extending lexicalized TAG to discourse. Cognitive Science, 28(5), 751-779.

Wolf, F. \& Gibson, E. (2006). Coherence in Natural Language: Data Structures and Applications. London: The MIT Press.

1. Néanmoins, Delaveau (1988) mentionne certains verbes de la Classe 3, par exemple commencer, continuer, enchaîner, interrompre, poursuivre, reprendre.

2. Ils modélisent en HPSG l'extraction de l'objet avec un trait slash dans l'incise, qui est considérée comme un ajout dans un schéma tête-ajout.

3. Les seuls arguments avancés par Lamiroy \& Charolles (2008, p. 8) pour considérer un verbe intransitif tel que plaisanter comme un transitif «mou »sont que la citation répond à la question en qu'est-ce que et que l'incise ne peut pas comporter d'objet direct. D'une part, ils illustrent ces tests uniquement sur le verbe dire, ce qui est pour le moins surprenant. D'autre part, il est faux d'affirmer qu'une incise de citation ne peut pas comporter d'objet direct, 
voir l'incise l'interrompit-il discutée ci-dessous. Signalons que Bonami \& Godard (2008) considèrent aussi qu'une incise de citation ne peut pas comporter d'objet direct.

4. Il est fait usage dans certains de ces exemples et des suivants de conventions typographiques différentes des guillemets, et notamment du tiret introducteur de tour de parole, qui n'indique pas explicitement la fin de la citation.

5. Nous avons toutefois trouvé un exemple où le syntagme nominal réfère aux propos interrompus (8), mais il nous semble inacceptable.

(8) — Ce n'est pas ça, interrompis-je le flot de ses paroles. [Vélimir Khlebnikov, traduit du russe par Luda Schnitzer, Le pieu du futur, p. 78]

6. Cet argument est de catégorie $\mathrm{P}$ si le connecteur apparaît en position initiale (Ensuite, Fred a décidé de partir), et de catégorie V s'il apparaît au milieu du noyau verbal ( Fred a ensuite décidé de partir).

7. Dans la perspective de la compréhension de textes, des ébauches d'interface phrase-discours sont présentées par Webber (2004) dans le cadre du formalisme D-LTAG et par Danlos (2009) dans le cadre du formalisme D-STAG.

8. Notons que ces motivations sont également valables pour les verbes de la Classe 3 étudiés précédemment, en plus des arguments déjà avancés.

9. La seule façon de faire allusion à l'intervention consiste à insérer un adverbe comme grossièrement : (22) a une lecture disant que l'intervention du député était grossière.

(22) Le député est intervenu grossièrement dans la discussion.

10. Ils indiquent trois occurrences où coasser est construit avec un objet direct (deux pour croasser) sans préciser si cet objet direct est un GN ou une complétive. D'après leur texte, nous pensons qu'il s'agit d'un GN, comme dans L'agonisant croasse un lamento qui navre [Google].

11. Dans ce sens, expliquer permet que son sujet soit non humain, voir (26).

(26) Des spéculations outrancières expliquent la hausse du prix du riz.

12. Ces auteurs diffèrent sur la nature « coordonnante » ou « subordonnante » de la relation Attribution, mas nous n'entrerons pas dans ce débat ici.

13. Si l'incise de citation comporte des ajouts, leur représentation sémantique est incluse dans $W$.

14. Les exemples en (40a-b) constituent un autre argument pour dire que la citation en (40b) n'est pas lié à l'objet direct de réclamer tel qu'il apparaît en (40a). En effet, la modalité est interdite dans la complétive et obligatoire dans la citation (39a-b).

(39) a. *Le député a réclamé que le gouvernement doive démissionner.

b. *"Le gouvernement démissionne", a réclamé le député. 